\title{
Molecular Mechanisms of Cardioprotective Actions of Tanshinones
}

\author{
Hyou-Ju Jin ${ }^{1,2}$ and Chun-Guang $\mathrm{Li}^{1}$ \\ ${ }^{1}$ National Institute of Complementary Medicine, Western Sydney University, Penrith, NSW 2751, Australia \\ ${ }^{2}$ Division of Chinese Medicine, School of Health Sciences, RMIT University, Bundoora, VIC 3083, Australia
}

Correspondence should be addressed to Chun-Guang Li; c.li@westernsydney.edu.au

Received 22 October 2015; Accepted 17 January 2016

Academic Editor: Hani El-Nezami

Copyright (C) 2016 H.-J. Jin and C.-G. Li. This is an open access article distributed under the Creative Commons Attribution License, which permits unrestricted use, distribution, and reproduction in any medium, provided the original work is properly cited.

Tanshinones are lipophilic compounds derived from Salvia miltiorrhiza (Danshen) that has been widely used to treat coronary heart diseases in China. The cardioprotective actions of tanshinones have been extensively studied in various models of myocardial infarction, cardiac ischemia reperfusion injury, cardiac hypertrophy, atherosclerosis, hypoxia, and cardiomyopathy. This review outlines the recent development in understanding the molecular mechanisms and signaling pathways involved in the cardioprotective actions of tanshinones, in particular on mitochondrial apoptosis, calcium, nitric oxide, ROS, TNF- $\alpha$, PKC, $\mathrm{PI} 3 \mathrm{~K} / \mathrm{Akt}$, IKK/NF- $\kappa \mathrm{B}$, and TGF- $\beta 1 /$ Smad mechanisms, which highlights the potential of these compounds as therapeutic agents for treating cardiovascular diseases.

\section{Introduction}

Danshen, also called red sage root, is dried roots of Salvia miltiorrhiza that is cultivated widely in many Asian countries including China, Korea, and Japan. Danshen and Danshen related products have long been used as traditional Chinese medicine to treat cardiovascular dysfunctions such as angina pectoris, stroke, and hypertension [1-4]. For example, a recent systematic review indicates that Danshen dripping pill is more effective than isosorbide dinitrate in treating angina pectoris [5]. Although with its long history of medicinal use, the mechanism of actions of Danshen has only been intensively investigated in recent years, with the progress in elucidating its phytochemistry and active compounds. The identified chemical constituents of Danshen can be classified into lipophilic and hydrophilic components. The main lipophilic constituents are tanshinones which are diterpene quinones. There are more than 40 tanshinones that have been isolated [6]. The main tanshinones include tanshinone I (TI), tanshinone IIA (TIIA), tanshinone IIB (TIIB), tanshinone VI (TVI), cryptotanshinone (CT), and dihydrotanshinone (DHT) as illustrated in Figure 1.
Tanshinones have been demonstrated with various pharmacological activities including antioxidant, anti-inflammatory, antibacterial, antineoplastic, immunomodulatory, cardioprotective, and neuroprotective actions and have been used in treating various conditions, including cardiovascular diseases, cancer, and diabetes [7-14]. One of the promising therapeutic actions of tanshinones is their cardioprotective actions [15]. For example, TIIA has been shown to dilate coronary vessels [16] and regulate vascular endothelial functions [17] and inhibit myocardial infarction [18], ischemia reperfusion (I/R) injury [19, 20], and cardiomyopathy [21]. It also reduced atherosclerosis [22]. CT has also been shown to reduce atherosclerosis and I/R induced cardiac injuries [23, 24], potentially via vasodilatation [25] and anti-inflammatory actions [26]. Similarly, TVI has been shown with antihypertrophy activity in cardiomyocytes [27], and DHT has been reported with vasorelaxation and antiplatelet actions $[28,29]$. Tables 1 and 2 summarize cardioprotective actions of major tanshinones in vitro and in vivo. This short review outlines the recent development in understanding the molecular mechanisms of cardioprotective actions of tanshinones, with a focus on their regulation of various signaling pathways. 
<smiles>Cc1coc2c1C(=O)C(=O)c1c-2ccc2c(C)cccc12</smiles>

Tanshinone I<smiles>Cc1cccc2c3c(ccc12)C1=C(C(=O)C3=O)C(C)CO1</smiles>

Dihydrotanshinone<smiles>Cc1coc2c1C(=O)C(=O)c1c-2ccc2c1CCCC2(C)C</smiles>

Tanshinone IIA<smiles>CC1COC2=C1C(=O)C(=O)c1c2ccc2c1CCCC2(C)C</smiles>

Cryptotanshinone<smiles>Cc1coc2c1C(=O)C(=O)c1c-2ccc2c1CCCC2(C)CO</smiles>

Tanshinone IIB<smiles>Cc1cccc2c3c(ccc12)C(O)=C(C(C)CO)C(=O)C3=O</smiles>

Figure 1: Chemical structures of major tanshinones.

\section{Effects of Tanshinones on Atherosclerosis}

Atherosclerosis is a vascular endothelial dysfunction caused by inflammation promoted by low-density lipoproteins (LDL) [30]. Various atherogenic stimuli, including diabetes and oxidative stress, can induce endothelial dysfunction, leading to atherosclerosis [31]. Current approaches for reducing atherosclerosis have been focused on antiinflammatory, antioxidant, and vasodilator mechanisms [32]. Studies have demonstrated the antiatherosclerosis actions of various tanshinones and their associations with various signaling pathways. For example, a tanshinone mixture was shown to inhibit proliferation of vascular smooth muscle cells (VSMCs) in vitro, which is associated with reduction of extracellular signal-regulated kinase 1/2 (ERK1/2) phosphorylation, cyclin D1, and enhancing $\mathrm{p} 21^{\text {waf1/cip1 }}$ (cyclindependent kinase inhibitor 1) expressions [33]. This is supported by the finding of inhibition of intimal hyperplasia by tanshinone in the ligated mouse carotid artery in vivo [34]. Treatment with total tanshinone reduced the development of intimal thickening of injured vessels and proliferating cell nuclear antigen- (PCNA-) positive vascular smooth muscle cells in mice, indicating that tanshinone may potentially inhibit VSMCs proliferation from arterial injury and reduce occurrence of atherosclerosis [34].

TIIA has been shown to increase nitric oxide (NO) generation and inhibiting platelet aggregation/leukocyte adhesion in vitro [73]. It also attenuated $\mathrm{H}_{2} \mathrm{O}_{2}$ induced endothelial dysfunction by enhancing superoxide dismutase (SOD) activity [38] and reducing caspase 3 activity in human umbilical vein endothelial cell (HUVECs) [39]. The antiatherosclerosis action of TIIA was associated with its anti-inflammatory activities by inhibition of cluster of differentiation 40 (CD40) and p53 protein expression [38, 39], and inhibition of tumor necrosis factor- $\alpha$ (TNF- $\alpha$ ) induced expressions of vascular cell adhesion molecule-1 (VCAM-1), intracellular adhesion molecule-1 (ICAM-1), and fractalkine, possibly through inhibition of IKK/NF- $\kappa$ B signaling pathway [37, 74]. TIIA was found to reduce oxidized LDL protein production and superoxide production by enhancing $\mathrm{Cu} / \mathrm{Zn} \mathrm{SOD}$ in rat [70] and also decreased cholesterol content and oxLDL level in apolipoprotein $\mathrm{E}$ deficient $\left(\mathrm{ApoE}^{-/-}\right)$mice [22]. It also inhibited the expression of inflammatory regulator CD40 and matrix metalloproteinase-2 (MMP-2) in high fat diet induced atherosclerosis in rabbits [71]. A recent study in ovariectomized $\mathrm{ApoE}^{-/-}$mice found that TIIA had a phytoestrogenlike activity reducing aortic lipid deposition and serum levels of malonyl dialdehyde (MDA), nuclear factor-kappa B (NF$\kappa \mathrm{B})$, and lipids, involving inhibiting p-ERK 1/2 [75]. Further study is required to elucidate the role of $\mathrm{NO}$ synthesis and redox signaling (specifically on regulation of mitochondrial anti/prooxidative stress) in the actions of TIIA.

Similarly, CT was found to attenuate TNF- $\alpha$ induced endothelial dysfunction in HUVECs by reducing endothelin1 accumulation, enhancing eNOS/NO, and inhibiting of NF$\kappa \mathrm{B}$ pathways [26]. In human aortic smooth muscle cells (HASMCs), CT inhibited TNF- $\alpha$ induced activation of NF$\kappa \mathrm{B}$ and activator protein 1 (AP1, a transcriptional factor that regulates genes of cytokines and growth factors), as well as activation of matrix metalloproteinases-9 (MMP-9), which plays an important role in migration and proliferation of vascular smooth cells and into the intima [23]. On the other hand, CT was found to reduce calcium flux and calcium induced contraction in rat coronary artery [25] and inhibit thromboxane A2 analogue U46619-induced constriction of the porcine coronary artery [76]. 


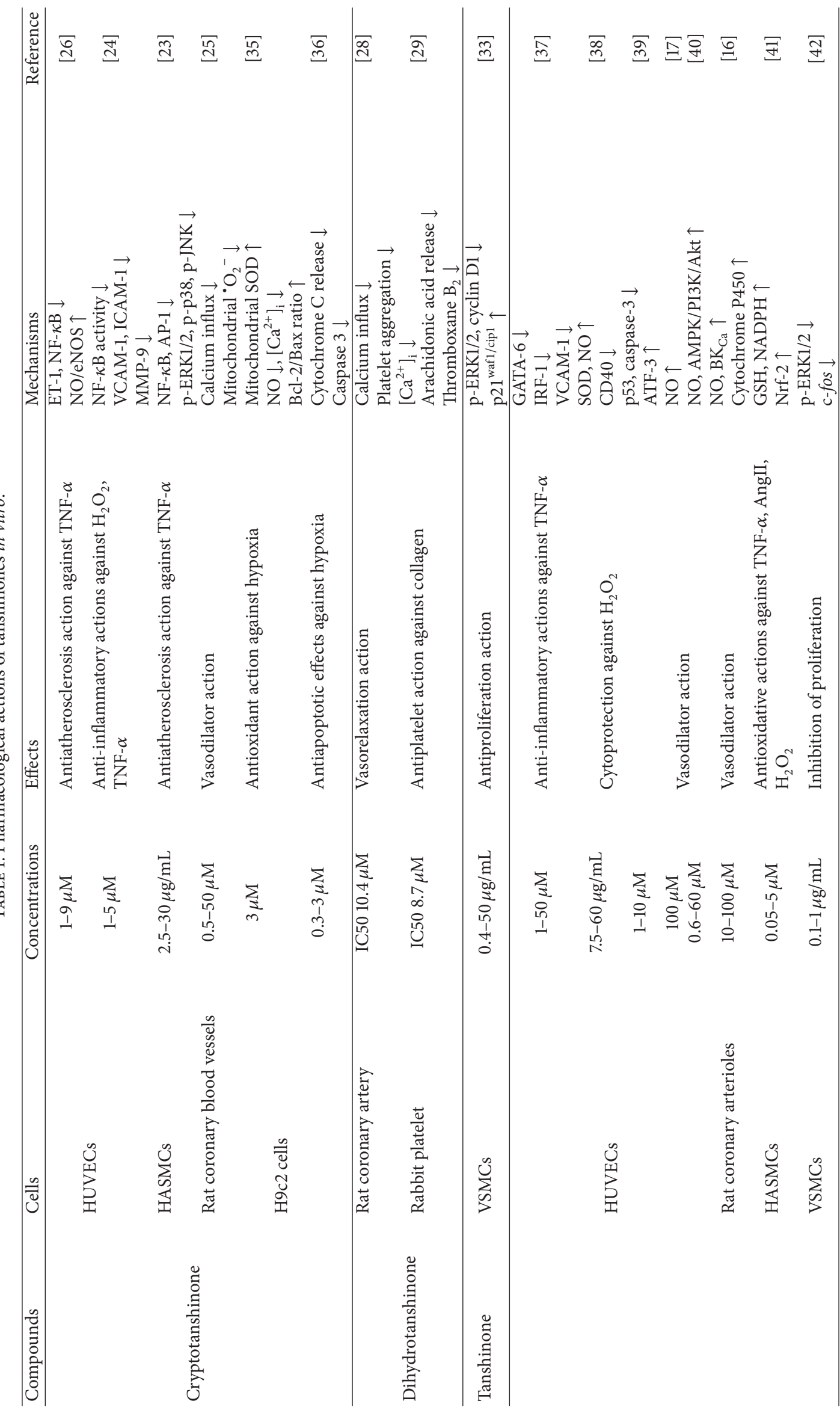




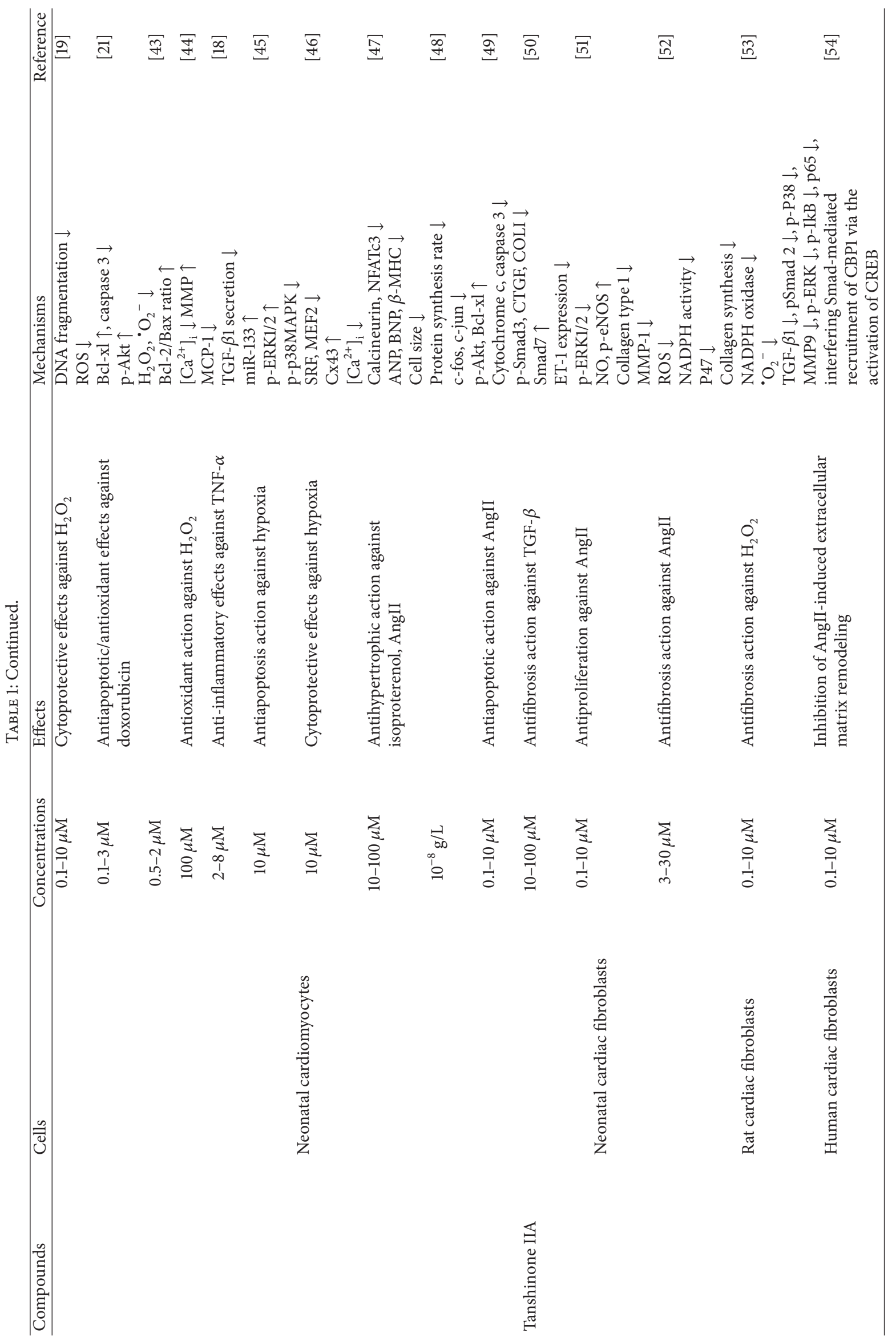




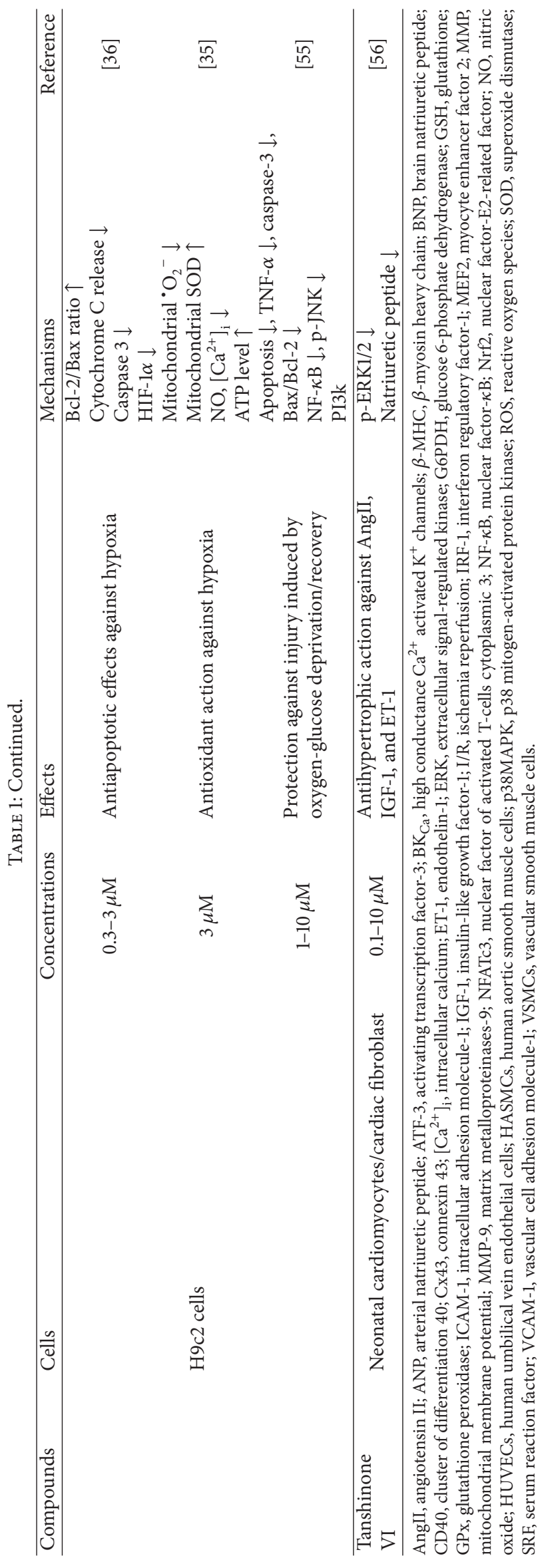


TABle 2: Pharmacological actions of tanshinones in vivo.

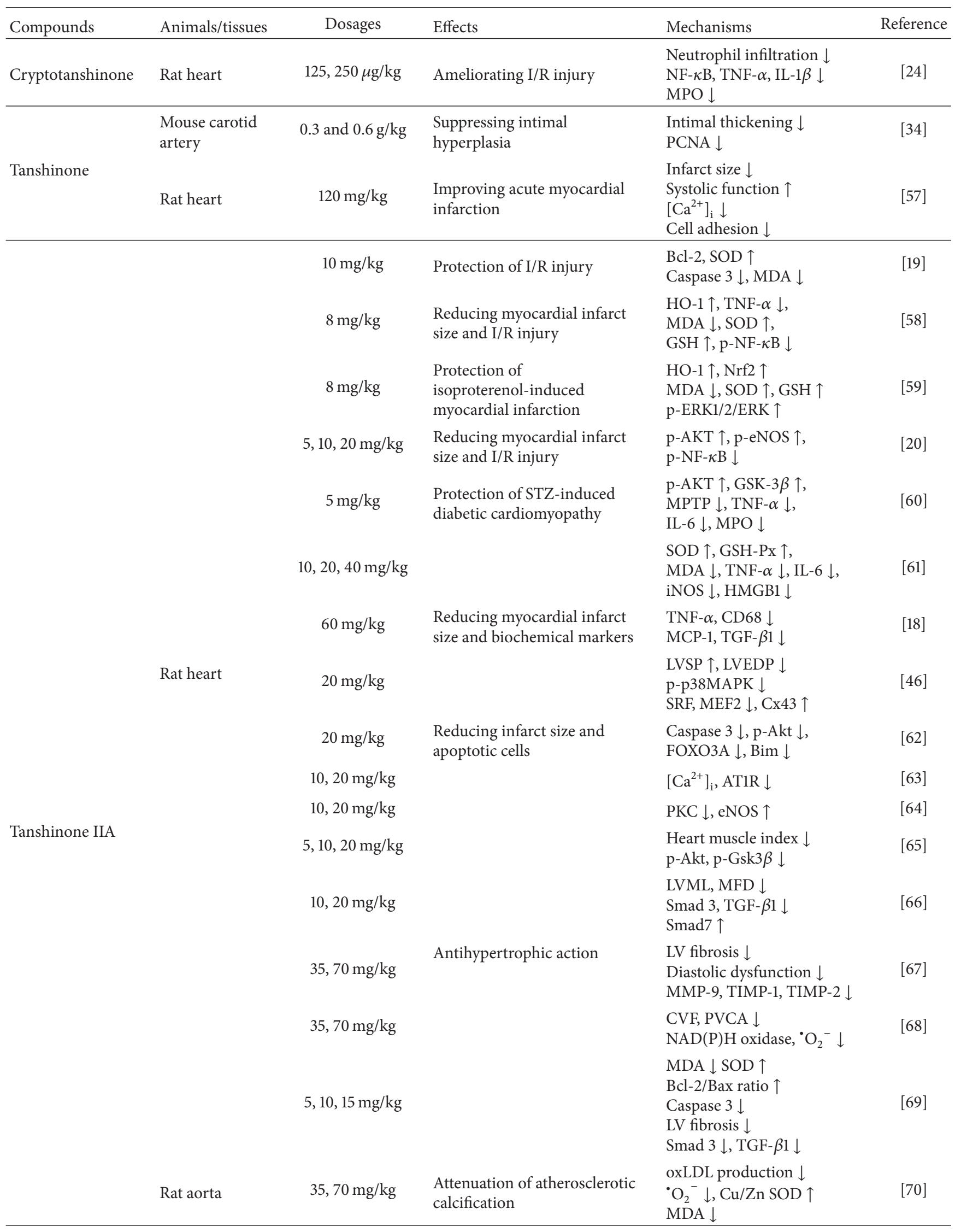


TABLE 2: Continued.

\begin{tabular}{|c|c|c|c|c|c|}
\hline Compounds & Animals/tissues & Dosages & Effects & Mechanisms & Reference \\
\hline & Mouse aorta & $10,30,90 \mathrm{mg} / \mathrm{kg}$ & Antiatherosclerotic action & $\begin{array}{l}\text { Cholesterol level } \downarrow \\
\text { CD36, SRA, PPAR } \gamma \downarrow\end{array}$ & {$[22]$} \\
\hline & Mouse heart & $20 \mathrm{mg} / \mathrm{kg}$ & Amelioration of I/R injury & $\begin{array}{l}\text { eNOS/NO } \uparrow \\
\text { AMPK/PI3K/Akt } \uparrow\end{array}$ & {$[40]$} \\
\hline & Rabbit aorta & $6.25,15.00,37.50 \mathrm{mg} / \mathrm{kg}$ & Antiatherosclerotic action & $\begin{array}{l}\text { CD40, MMP- } 2 \downarrow \\
\text { SOD } \uparrow\end{array}$ & {$[71]$} \\
\hline
\end{tabular}

AT1R, angiotensin II type I receptor; $\left[\mathrm{Ca}^{2+}\right]_{\mathrm{i}}$, intracellular calcium; CD36, cluster of differentiation 36; CD68, cluster of differentiation 68; CVF, collagen volume fraction; $\mathrm{Cx} 43$, connexin 43; Dox, doxorubicin; IL-1 $\beta$, interleukin-1 beta; IL-6, interleukin-6; I/R, ischemia reperfusion; LV: left ventricular; LVMI, left ventricular mass index; LVSP, left ventricular systolic pressure; LVEDP, left ventricular end diastolic pressure; MDA, malondialdehyde; MEF2, myocyte enhancer factor 2; MFD, myocardial fiber diameter; MI, myocardial infarction; MMP-2, matrix metalloproteinases-2; MPO, myeloperoxidase; NF- $\kappa$ B, nuclear factor-kappa B; PPAR $\gamma$, peroxisome proliferator activated gamma; PCNA, proliferating cell nuclear antigen; PVCA, perivascular collagen area; ROS, reactive oxygen species; SOD, superoxide dismutase; SRA, scavenger receptor A; SRF, serum reaction factor; TGF- $\beta 1$, transforming growth factor-beta 1.

Another tanshinone, DHT, was shown with a vasodilator activity by reducing $\mathrm{CaCl}_{2}$ elicited contraction in rat coronary artery [28]. The calcium regulation effect of DHT was also demonstrated in its inhibition of collagen-induced rabbit platelet aggregation, which involves intracellular calcium mobilization, arachidonic acid liberation, and thromboxane B2 generation mechanisms [29].

\section{Effects of Tanshinones on Myocardial Infarction}

Myocardial infarction (MI) is caused by disruption of blood supply to the heart and often accompanied by ventricular remodeling and cardiac hypertrophy, which can lead to cardiac cell death [77]. Cardiomyocytes apoptosis is an important mechanism in MI [78], and it is closely associated with cellular inflammatory reaction, intracellular calcium homeostasis, and NO and oxidative stress [79].

There is evidence that tanshinones reduced cardiac infarct size and improved cardiac function in MI via inhibiting intracellular calcium, cell adhesion molecules, apoptotic protein, and inflammation protein expressions. An in vivo study demonstrated that acute myocardial infarction induced by occlusion of rat coronary vessel was decreased by posttreatment of a tanshinone extract [57]. This improvement was partially mediated by modulation of expression of genes which regulate intracellular calcium, cell adhesion molecules, and alternative complementary pathways (i.e., neuronal pentraxin 1, intercellular adhesion molecule 1, and C3 convertase) $[46,57]$. The anti-inflammatory and antiapoptotic properties of tanshinones may play an important role in their anti-MI actions $[18,46]$. TIIA was shown to inhibit TNF- $\alpha$ induced monocyte chemoattractant protein-1 (MCP-1) and transforming growth factor- $\beta 1$ (TGF- $\beta 1$ ) expression in cardiac fibroblasts in vitro [18]. It reduced TNF- $\alpha$, NF- $\kappa \mathrm{B}, \mathrm{MCP}-1$, and TGF- $\beta 1$ expressions [18] and improved cardiac dysfunction with inhibition of expressions of $\mathrm{p}-38$, serum response factor (SRF: a transcription factor that has a role in modulating muscle proliferation and cell migration and apoptosis), and myocyte enhancer factor 2 (MEF2: a transcription factor that regulates tissue remodeling in cardiac muscle) [46].

\section{Effects of Tanshinones on Ischemia Reperfusion Injuries}

Myocardial ischemia reperfusion (I/R) injury is caused by insufficient oxygen supply to the cardiac tissue which can occur in various conditions, such as thrombosis, coronary atherosclerotic plaques, and diabetes complications [80]. Sudden oxygen supply during reperfusion after prolonged ischemia can lead to impairment of myocardial cells via reactive oxygen species ( $\mathrm{ROS}$ ) dependent mechanisms [81]. The myocardial I/R injury can be affected by a number of cellular factors/regulators including calcium, $\mathrm{pH}, \mathrm{ROS}$, and in particular mitochondrial function. For example, mitochondrial membrane transition pore (MPTP) opening has been a research focus and potential therapeutic target for developing new treatments for I/R injury in recent years [82]. I/R injuries are likely to be the main cause of MI and subsequently heart failure due to imbalance in redox signaling [83]. Therefore, regulating redox signaling during reperfusion may be an important turning point in treating MI and heart failure [84].

Tanshinones have been well demonstrated with protective actions on reducing ischemia I/R injuries involving inhibition of free radical formation and mitochondrial apoptosis, as well as regulating eNOS/NO and AMPK/PI3K/Akt pathways. In a rat model of I/R injury, TIIA $(20 \mathrm{mg} / \mathrm{kg})$ was shown to reduce infarction size, possibly by upregulating eNOS/NO pathway and AMPK/PI3K/Akt pathway [40]. TIIA was also shown to enhance glutathione peroxidase and SOD activities and decrease production of free radicals $\left(\mathrm{H}_{2} \mathrm{O}_{2}\right.$ and $\left.\mathrm{O}_{2}{ }^{-}\right)$ $[19,44,85]$. Since glutathione peroxidase, rather than SOD, is the major antioxidant in the cardiomyocytes [86], TIIA may be a promising agent to treat $\mathrm{I} / \mathrm{R}$ injuries and $\mathrm{MI}$ due to its better permeability to cell membranes.

TIIA has been shown to inhibit hydrogen peroxide induced cell apoptosis in neonatal cardiomyocytes in vitro, with a $40 \%$ reduction of cell death as evidenced by decreased Hoechst 33342 staining and DNA fragmentation [19]. Similar findings were reported for its inhibition of $\mathrm{H}_{2} \mathrm{O}_{2}$ induced cardiomyocytes cell apoptosis and intracellular calcium increase [44]. However, the exact role of TIIA in I/R injuries still needs to be elucidated due to its complex interactions of $\mathrm{H}_{2} \mathrm{O}_{2}$ with 
other signaling pathways. Additionally, antiapoptotic actions of TIIA have been shown in neonatal cardiomyocytes under hypoxia condition, with reducing p38 mitogen-activated protein kinase (p38MAPK) phosphorylation and miR-1 level [46], and enhanced ERK1/2 phosphorylation [45]. Our study has also demonstrated that TIIA $(3 \mu \mathrm{M})$ reduced hypoxia induced late apoptosis in $\mathrm{H} 9 \mathrm{C} 2$ cells, the effect linked to decreasing hypoxia inducible factor- $1 \alpha$ (HIF- $\alpha$ ) translocation, balancing in $\mathrm{Bcl}-2 / \mathrm{Bax}$ ratio, reducing cytochrome $\mathrm{c}$ release and caspase 3 activity [36]. A recent study also found that the protection by TIIA on hypoxic ischemia-induced injury could be enhanced by the JAK2/STAT3 inhibitors [87]. Additionally, the antiapoptosis effects of TIIA were associated with regulation of intracellular calcium, ATP, mitochondrial superoxide, and SOD levels but not intracellular $\mathrm{H}_{2} \mathrm{O}_{2}$ [35]. Studies in vivo found that TIIA inhibited I/R induced apoptosis (about $50 \%$ reduction of TUNEL staining), reduced caspase 3 activity, increased SOD level and Bcl-2/Bax ratio [19], and restored $\mathrm{I} / \mathrm{R}$ induced diminished eNOS/NO production and coronary dysfunction [40]. Thus, it is likely that the protection of TIIA on myocardial I/R injuries involves multiple mechanisms including inhibition of intrinsic apoptosis pathway and anti-inflammatory and antioxidant activities. Interestingly, recent studies on cerebral I/R injury also indicate that TIIA may act by inhibiting intrinsic death pathway, inflammatory response, and ROS [7, 88].

Similarly, CT was shown to reduce hypoxia induced $\mathrm{H} 9 \mathrm{c} 2$ cell apoptosis by balancing $\mathrm{Bcl}-2 / \mathrm{Bax}$ ratio, reducing hyperpolarisation in mitochondria membrane potential and caspase-3 activity [36]. Additionally, CT prevented the hypoxia induced changes of intracellular calcium, mitochondrial SOD, superoxide level, and NO [35]. A study in I/R model in vivo demonstrated that CT reduced myocardial infarction and inhibited proinflammatory cytokines including TNF- $\alpha$, IL-1 $\beta$ (interleukin-1 beta), and IL-6 (interleukin6) production, NF- $\kappa \mathrm{B}$ translocation, and neutrophil infiltration [24].

TVI has also been demonstrated with protective action on hypoxia/reoxygenation injury and improvement of cardiac function [89]. TVI treatment improved myocardial contractility in reoxygenated rat heart, accompanied by reduced release of ATP metabolites and decrease in cellular calcium contents, but further study is required to elucidate its actions on ROS mechanisms [89].

\section{Effects of Tanshinones on Cardiac Hypertrophy}

Cardiac hypertrophy is defined as an enlargement of cardiac tissue or myocardium, resulting in a decrease in size of ventricle chambers. The pathological cardiac hypertrophy is associated with increased interstitial fibrosis, cell death, and cardiac dysfunction and influenced by many factors/conditions such as hypertension, ischemic injury, and genetic polymorphism $[90,91]$. The main cellular and molecular regulators of cardiac hypertrophy include calcineurin/nuclear factor of activated T-cells (NFAT), G protein-coupled receptors (GPCR), MAPKs and apoptosis pathways, and cytokines activated by various stress stimuli including AngII, endothelin-1, and insulin-like growth factor-1 (IGF-1) [91, 92]. Pathological cardiac hypertrophy has been shown to be more susceptible to proapoptotic changes, including modulations of Fas, Bcl2 family proteins and caspases [93], and apoptosis occurring during hypertrophy may act as a cardiac adaptive response [94, 95]. Additionally, hypertrophic stimuli (i.e., angiotensin II (AngII) and endothelin-1 (ET-1) and catecholamines) have also been shown to induce hypertrophy by stimulating ROS production (via NADPH oxidase, xanthine oxidase, and nitric oxide synthase) [96]. It has been suggested that NADPH oxidase plays an importance role regarding redoxsignaling involvement in pathological hypertrophy [92].

The anticardiac hypertrophy actions of tanshinones have been demonstrated in vitro and in vivo (see Tables 1 and 2). TIIA inhibited the size of enlarged cell induced by isoproterenol [47] and AngII [48, 49] and inhibited mRNA expressions of c-fos, c-jun (immediate-early genes of hypertrophic markers) induced by AngII [48]. The antiapoptotic actions of TIIA were shown via enhancing Bcl-xl expression and Akt phosphorylation, decreasing apoptotic markers (cytochrome c protein, cleaved caspase 3 levels) and reducing ROS production [49]. Furthermore, TIIA inhibited isoproterenolinduced increase in intracellular calcium level and reduced calcineurin/nuclear factors derived from activated T-cells cytoplasmic 3 (NFATc3) proteins expression [47]. TIIA has also been shown with antifibrosis effects by reducing TGF$\beta$ [50]/hydrogen peroxide [53] induced collagen synthesis in cardiac fibroblasts. The antifibrosis of TIIA may involve regulating Smad proteins expression (increasing Smad-7 and decreasing p-Smad 3) [50] and $\mathrm{NAD}(\mathrm{P}) \mathrm{H}$ oxidase activity (reducing Nox2, Nox4, and p47phox protein levels) [53]. The antihypertrophy actions of tanshinones have been confirmed in vivo in various animal models including those by abdominal aorta constriction $[63,64,66]$, thoracic aorta partial constriction [65], and two-kidney-two-clip hypertensive rats $[67,68]$. The main finding from in vivo studies is that TIIA reduced left ventricular mass index but did not alter blood pressure. The actions of TIIA may involve its interactions with various signaling pathways including protein kinase $\mathrm{C}(\mathrm{PKC})$, calcium, phosphoinositide 3-kinase (PI3K)/Akt (reducing p-Akt and p-Gsk3 $\beta$ ), TGF- $\beta 1 /$ Smad (decreasing Smad 3, TGF- $\beta 1$ and increasing Smad 7), and oxidant signaling. TIIA has been shown to inhibit intracellular calcium [63], PKC [64] and its downstream c-fos/c-jun [48], and matrix metalloproteinases 9 (MMP9)/tissue inhibitor of metalloproteinases type 1 (TIMP1) [67]. However, it is still not clear how TIIA interacts with calcineurin/NFAT proteins (downstream of calcium signaling) and GPCR (upstream of calcium signaling). Furthermore, it has been suggested that TIIA acts by regulating NADPH oxidase activity [92] and its subunits (decreasing Nox2, Nox4, and p47phox proteins levels) [68] and inhibiting eNOS-mediated endothelial dysfunctions [64].

Other tanshinones, such as TVI, have also been shown with antihypertrophy actions in neonatal cardiomyocytes/ fibroblasts induced by IGF-1, AngII, and ET-1 [56, 89]. TVI inhibited IGF-1-induced cardiomyocyte hypertrophy and fibrosis of cardiac fibroblasts and collagen synthesis in vitro [89]. Further study found that TVI attenuated 


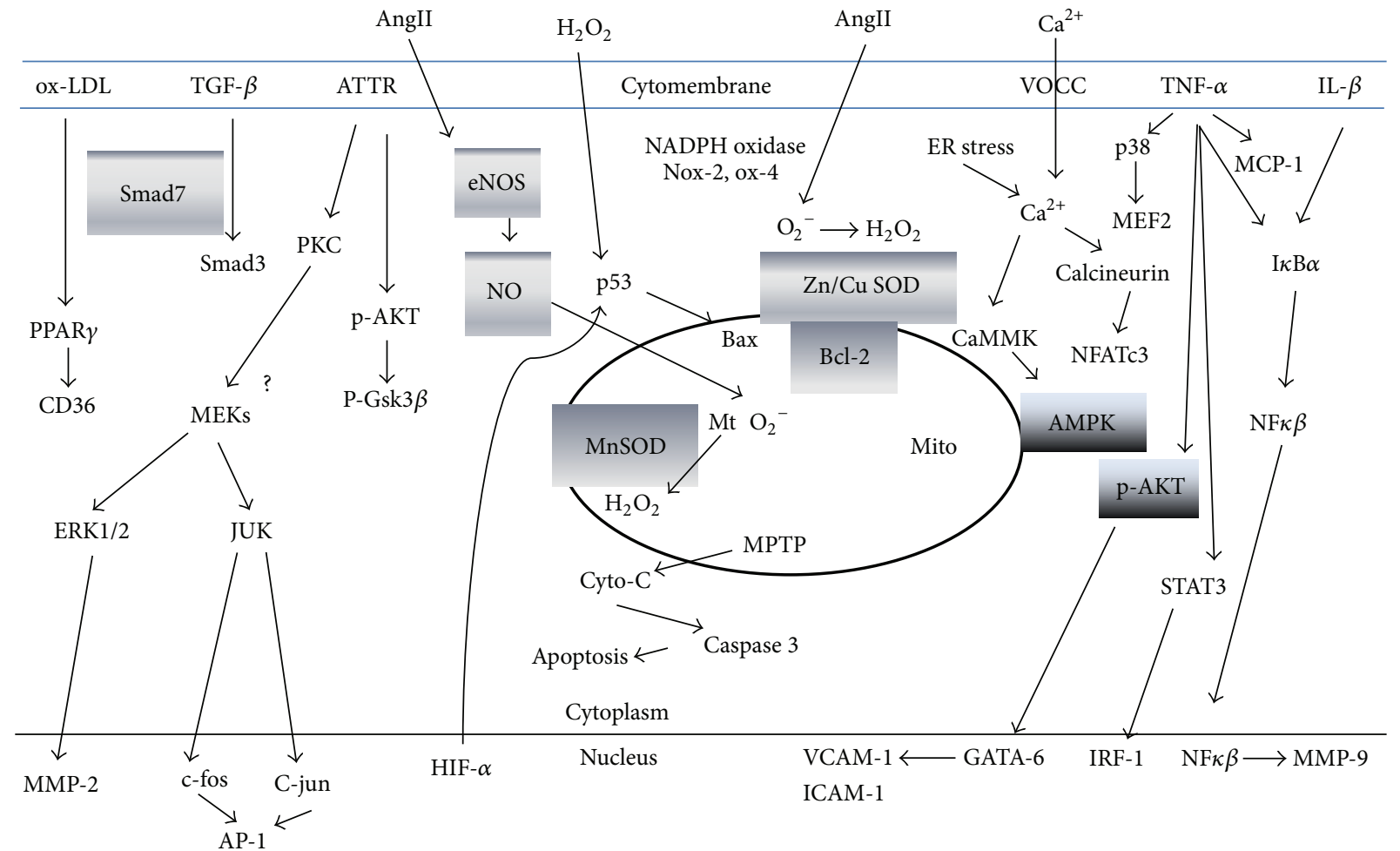

FIGURE 2: Molecular targets in cardioprotective actions of tanshinones. This schematic illustration summarizes the mechanisms involved in cardioprotective actions of tanshinones based on studies on TIIA and CT. The targets with amelioration by tanshinones are indicated with the shaded text box. The targets with attenuation by tanshinones are indicated without shaded text box. Main cardioprotective actions of tanshinones were characterized via anti-inflammatory, antioxidant, antiapoptosis, antiproliferation/fibrosis, and vasodilator actions. The anti-inflammatory actions of tanshinones are via inhibition of TNF- $\alpha$ and IL- $\beta$ and their downstream signaling pathways including IkB $\alpha / \mathrm{NF}$ $\kappa \mathrm{B}, \mathrm{MCP}-1, \mathrm{STAT} 3$, and Akt, resulting in reduced IRF-1 and GATA-6 proteins expression which in turn decreases VCAM-1. ERK1/2, p38, and JNK are also inhibited. By suppressing ERK1/2 and JNK phosphorylation, CT reduces AP-1 activation. However, it is undetermined whether CT inactivates AP-1 via reduction of $c-$ fos and c-jun. The inhibition of NF- $\kappa$ B's translocation to nucleus results in decreased MMP-9 expression. Furthermore, TIIA decreases PPAR $\gamma$ expression and CD36 which inhibits cell formation and subsequently inflammation. TIIA exerts antioxidant effects by enhancing antioxidant enzymes ( $\mathrm{Cu} / \mathrm{Zn} \mathrm{SOD}, \mathrm{Mn}-\mathrm{SOD}$, and $\mathrm{GSH})$ and decreasing intracellular $\mathrm{H}_{2} \mathrm{O}_{2} / \mathrm{O}_{2}{ }^{-}$, mitochondrial $\mathrm{O}_{2}^{-}$levels, and Nox 2 and Nox 4. The antiapoptosis action of tanshinones is via balancing Bcl-2/Bax protein, reducing cytochrome $\mathrm{C}$ release, and inhibiting caspase 3 activation. Bax can be activated by p53 which can be activated by HIF-1 $\alpha$. TIIA inhibits HIF- $1 \alpha$ translocation to nucleus which in turn could reduce p53-induced Bax activation and ultimately apoptosis. Tanshinones inhibit the apoptosis by reducing mitochondrial superoxide level and enhancing Mn-SOD, increasing NO level, and decreasing intracellular calcium level. Antiproliferation/fibrosis actions of TIIA involve calcineurin-NFAT pathway, TGF- $\beta /$ Smad pathway, PKC (ERK1/2 and JNK) pathway, and Akt/Gsk3 $\beta$ signaling. As results of ERK1/2 and JNK inhibition by TIIA, c-fos and c-jun were inhibited by TIIA as well as MMP2. Additionally, tanshinones present vasodilator action by reducing calcium influx, regulating intracellular calcium and NO levels, which may also involve inhibition of ER stress. AMPK, adenosine monophosphate kinase; AP-1, activator protein-1; AT1R, angiotensin II type 1 receptor; Cyto-C, cytochrome C; Casp 3, caspase 3; CD36, cluster of differentiation 36; Cu/Zn SOD, copper/zinc superoxide dismutase; eNOS, endothelial nitric oxide synthase; ERK, extracellular signal-regulated kinase; GSH, glutathione; Gsk-3 $\beta$, glycogen synthase kinase-3 beta; HIF$1 \alpha$, hypoxia inducible factor-1 alpha; ICAM-1, intracellular adhesion molecule-1; IL-1 $\beta$, interleukin-1 beta; JNK, c-Jun N-terminal kinases; MCP-1, monocyte chemotactic protein-1; MEKs, MAPK/ERK kinases; MMP-2, matrix metallopeptidase-2; MMP-9, matrix metallopeptidase-

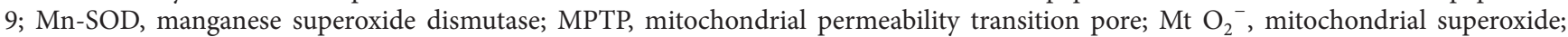
NADPH, nicotinamide adenine dinucleotide phosphate; NFATc3, nuclear factor of activated T-cells, cytoplasmic 3; NO, nitric oxide; Nox 2, NADPH oxidase 2; Nox 4, NADPH oxidase 4; PKC, protein kinase C; STAT3, signal transducer and activator of transcription 3; TNF- $\alpha$, tumor necrosis factor-alpha; VCAM-1, vascular cell adhesion molecule-1.

$\left[{ }^{3} \mathrm{H}\right]$-leucine incorporation, ERK1/2 phosphorylation, and atrial natriuretic peptide level but not Akt phosphorylation [56]. However, the actions of TVI on upstream signaling, which regulates ERK1/2 phosphorylation, such as GPCR, ROS, and NADPH oxidase, are still not clear.
In addition, there is evidence for the involvement of microRNAs in the cardioprotection of tanshinones. It was found that TIIA increased the expressions of MiR-133 and ERK1/2 in hypoxic neonatal cardiomyocytes [45]. In rats with acute myocardial infarction, TIIA inhibited postinfarct 
cardiac fibrosis and improved impaired left ventricular function, with downregulation of expression of TGF- $\beta 1$ and upregulation of expression of miR-29b. The antifibrotic effect of TIIA was blocked by a miR-29b inhibitor and Smad3 siRNA, indicating that miR-29 may play an important role in the action of TIIA in postinfarct cardiac remodeling, possibly mediated by TGF- $\beta$-Smad3 signaling pathway [97].

\section{Effects of Tanshinone IIA on Adriamycin Induced Cardiomyopathy}

Adriamycin (ADR), also called doxorubicin, is an effective anticancer drug. However, its clinical usage is limited by its cardiomyopathy which may lead to heart failure [98]. ADRinduced cardiomyopathy is related to increased oxidative stress [99]. Increasing ROS can reduce mitochondria oxidative phosphorylation, which in turn depletes cellular ATP, resulting in cardiac cell impairment [100]. Other possible mechanisms for ADR-induced cardiomyopathy include activation of mitogen-activated protein kinases (MAPKs) and necrosis and extrinsic pathways [101]. TIIA has been demonstrated to prevent ADR-induced cardiomyocytes death in vitro by preventing DNA fragmentation (60\% compared to positive control), suppressing ROS production, and increasing $\mathrm{Bcl}-2 / \mathrm{Bax}$ ratio [43]. Hong et al. also showed that TIIA prevented ADR-induced cell apoptosis and ROS production by $33 \%$ and $37 \%$, respectively, with enhanced $\mathrm{Bcl}-\mathrm{xl}$ protein expression, inhibited cytochrome $c$ release and caspase 3 activity, and restored Akt phosphorylation [21]. Thus, TIIA and other tanshinone analogues may potentially be useful in preventing ADR-induced cardiomyopathy.

\section{Effects of Tanshinone IIA on Endotoxin-Induced Cardiac Dysfunction}

Tanshinone IIa has recently been shown to attenuate endotoxin-induced cardiac dysfunction in septic mice via inhibiting NADPH oxidase 2-related signaling pathway. It inhibited LPS-induced production of TNF- $\alpha$, IL- $1 \beta$, and ROS and decreased Nox2, p-ERK1/2, and p38 MARK [72].

\section{Synthetic Tanshinone Derivatives and Structure Optimization}

Natural tanshinones have a unique structure and bioactivity but also some limitations such as low water solubility and poor bioavailability [12]. Approaches have been made to develop synthetic derivatives of tanshinones to optimize their structures for potential clinical applications. For example, sodium tanshinone IIA sulfonate was synthesized as a water soluble derivative of tanshinone IIA, which has been used in China for the treatment of angina pectoris and myocardial infarction [102]. Several tanshinone IIA derivatives with better vasodilatation activity have been synthesized by the reactions of tanshinone IIA with aromatic aldehydes, and the structure-activity relationship indicated that the methyoxylsubstituting group in the meta-position of aromatic aldehydes presented better activity than that of nitro substitution derivatives [103]. A number of tanshinone derivatives have been synthesized with modification of tanshinones' four rings with fatty acids, N-containing substituent, and other function groups, resulting in analogues with potential better bioactivity and bioavailability [102].

\section{Conclusion}

In conclusion, tanshinones have been demonstrated with significant cardiac protective actions, which may be potentially useful as therapeutic agents to treat cardiac diseases. The molecular mechanisms of cardioprotective actions of tanshinones involve anti-inflammatory, antioxidant, and antiapoptosis actions although regulating various signaling pathways, in particular TNF-alpha signaling, mitochondrial apoptosis, and calcium and NO signaling pathways, as illustrated in Figure 2. Further studies on tanshinones and tanshinone analogues may lead to development of novel drugs for treating cardiovascular diseases.

\section{Conflict of Interests}

The authors declare that there is no conflict of interests regarding the publication of this paper.

\section{Acknowledgment}

This work was supported by grants from Western Sydney University.

\section{References}

[1] L. Zhou, Z. Zuo, and M. S. S. Chow, "Danshen: an overview of its chemistry, pharmacology, pharmacokinetics, and clinical use," Journal of Clinical Pharmacology, vol. 45, no. 12, pp. 1345-1359, 2005.

[2] B. Wu, M. Liu, and S. Zhang, "Dan Shen agents for acute ischemic stroke," Stroke, vol. 38, no. 11, pp. 3095-3096, 2007.

[3] T. O. Cheng, "Danshen: a versatile Chinese herbal drug for the treatment of coronary heart disease," International Journal of Cardiology, vol. 113, no. 3, pp. 437-438, 2006.

[4] J. D. Adams, R. Wang, J. Yang, and E. J. Lien, "Preclinical and clinical examinations of Salvia miltiorrhiza and its tanshinones in ischemic conditions," Chinese Medicine, vol. 1, article 3, 2006.

[5] Y. Jia, F. Huang, S. Zhang, and S.-W. Leung, "Is danshen (Salvia miltiorrhiza) dripping pill more effective than isosorbide dinitrate in treating angina pectoris? A systematic review of randomized controlled trials," International Journal of Cardiology, vol. 157, no. 3, pp. 330-340, 2012.

[6] Y. Dong, S. L. Morris-Natschke, and K.-H. Lee, "Biosynthesis, total syntheses, and antitumor activity of tanshinones and their analogs as potential therapeutic agents," Natural Product Reports, vol. 28, no. 3, pp. 529-542, 2011.

[7] Y. Chen, X. Wu, S. Yu et al., "Neuroprotective capabilities of tanshinone IIA against cerebral ischemia/reperfusion injury via anti-apoptotic pathway in rats," Biological and Pharmaceutical Bulletin, vol. 35, no. 2, pp. 164-170, 2012.

[8] D. Wang, W. Zhang, T. Wang et al., "Unveiling the mode of action of two antibacterial tanshinone derivatives," International Journal of Molecular Sciences, vol. 16, no. 8, pp. 1766817681, 2015. 
[9] X. Wang, S. L. Morris-Natschke, and K.-H. Lee, "New developments in the chemistry and biology of the bioactive constituents of tanshen," Medicinal Research Reviews, vol. 27, no. 1, pp. 133148, 2007.

[10] J.-Y. Han, J.-Y. Fan, Y. Horie et al., "Ameliorating effects of compounds derived from Salvia miltiorrhiza root extract on microcirculatory disturbance and target organ injury by ischemia and reperfusion," Pharmacology and Therapeutics, vol. 117, no. 2, pp. 280-295, 2008.

[11] J.-Y. Han, Y. Horie, S. Miura et al., "Compound Danshen injection improves endotoxin-induced microcirculatory disturbance in rat mesentery," World Journal of Gastroenterology, vol. 13, no. 26, pp. 3581-3591, 2007.

[12] Y. Zhang, P. Jiang, M. Ye, S.-H. Kim, C. Jiang, and J. Lü, “Tanshinones: sources, pharmacokinetics and anti-cancer activities," International Journal of Molecular Sciences, vol. 13, no. 10, pp. 13621-13666, 2012.

[13] S. Xu and P. Liu, "Tanshinone II-A: new perspectives for old remedies," Expert Opinion on Therapeutic Patents, vol. 23, no. 2, pp. 149-153, 2013.

[14] Q. Liu, Y. Zhang, Z. Lin et al., "Danshen extract 15,16-dihydrotanshinone I functions as a potential modulator against metabolic syndrome through multi-target pathways," The Journal of Steroid Biochemistry and Molecular Biology, vol. 120, no. 4-5, pp. 155-163, 2010.

[15] Q. Shang, H. Xu, and L. Huang, "Tanshinone IIA: a promising natural cardioprotective agent," Evidence-Based Complementary and Alternative Medicine, vol. 2012, Article ID 716459, 7 pages, 2012.

[16] G.-B. Wu, E.-X. Zhou, and D.-X. Qing, “Tanshinone $\mathrm{II}_{\mathrm{A}}$ elicited vasodilation in rat coronary arteriole: roles of nitric oxide and potassium channels," European Journal of Pharmacology, vol. 617, no. 1-3, pp. 102-107, 2009.

[17] K.-J. Huang, H. Wang, W.-Z. Xie, and H.-S. Zhang, "Investigation of the effect of tanshinone IIA on nitric oxide production in human vascular endothelial cells by fluorescence imaging," Spectrochimica Acta-Part A: Molecular and Biomolecular Spectroscopy, vol. 68, no. 5, pp. 1180-1186, 2007.

[18] Z. H. Ren, Y. H. Tong, W. Xu, J. Ma, and Y. Chen, “Tanshinone II A attenuates inflammatory responses of rats with myocardial infarction by reducing MCP-1 expression," Phytomedicine, vol. 17, no. 3-4, pp. 212-218, 2010.

[19] J. Fu, H. Huang, J. Liu, R. Pi, J. Chen, and P. Liu, “Tanshinone IIA protects cardiac myocytes against oxidative stress-triggered damage and apoptosis," European Journal of Pharmacology, vol. 568, no. 1-3, pp. 213-221, 2007.

[20] X. Yuan, S. Jing, L. Wu, L. Chen, and J. Fang, "Pharmacological postconditioning with tanshinone IIA attenuates myocardial ischemia-reperfusion injury in rats by activating the phosphatidylinositol 3-kinase pathway," Experimental and Therapeutic Medicine, vol. 8, no. 3, pp. 973-977, 2014.

[21] H.-J. Hong, J.-C. Liu, P.-Y. Chen, J.-J. Chen, P. Chan, and T.-H. Cheng, "Tanshinone IIA prevents doxorubicin-induced cardiomyocyte apoptosis through Akt-dependent pathway," International Journal of Cardiology, vol. 157, no. 2, pp. 174-179, 2012.

[22] F.-T. Tang, Y. Cao, T.-Q. Wang et al., “Tanshinone IIA attenuates atherosclerosis in $\mathrm{ApoE}^{-/-}$mice through down-regulation of scavenger receptor expression," European Journal of Pharmacology, vol. 650, no. 1, pp. 275-284, 2011.
[23] S.-J. Suh, U.-H. Jin, H.-J. Choi et al., "Cryptotanshinone from Salvia miltiorrhiza BUNGE has an inhibitory effect on TNF- $\alpha$ induced matrix metalloproteinase- 9 production and HASMC migration via down-regulated NF- $\kappa \mathrm{B}$ and AP-1," Biochemical Pharmacology, vol. 72, no. 12, pp. 1680-1689, 2006.

[24] Y. C. Jin, C. W. Kim, Y. M. Kim et al., "Cryptotanshinone, a lipophilic compound of Salvia miltiorrriza root, inhibits TNF$\alpha$-induced expression of adhesion molecules in HUVEC and attenuates rat myocardial ischemia/reperfusion injury in vivo," European Journal of Pharmacology, vol. 614, no. 1-3, pp. 91-97, 2009.

[25] F. F. Y. Lam, J. H. K. Yeung, K. M. Chan, and P. M. Y. Or, "Mechanisms of the dilator action of cryptotanshinone on rat coronary artery," European Journal of Pharmacology, vol. 578, no. 2-3, pp. 253-260, 2008.

[26] Z. Zhou, S.-Q. Wang, Y. Liu, and A.-D. Miao, "Cryptotanshinone inhibits endothelin-1 expression and stimulates nitric oxide production in human vascular endothelial cells," Biochimica et Biophysica Acta (BBA)_General Subjects, vol. 1760, no. 1, pp. 1-9, 2006.

[27] T. Maki, Y. Kawahara, K. Tanonaka, A. Yagi, and S. Takeo, "Effects of tanshinone VI on the hypertrophy of cardiac myocytes and fibrosis of cardiac fibroblasts of neonatal rats," Planta Medica, vol. 68, no. 12, pp. 1103-1107, 2002.

[28] F. F. Y. Lam, J. H. K. Yeung, K. M. Chan, and P. M. Y. Or, "Dihydrotanshinone, a lipophilic component of Salvia miltiorrhiza (danshen), relaxes rat coronary artery by inhibition of calcium channels," Journal of Ethnopharmacology, vol. 119, no. 2, pp. 318-321, 2008.

[29] J.-W. Park, S.-H. Lee, M.-K. Yang et al., “15,16-Dihydrotanshinone I, a major component from Salvia miltiorrhiza Bunge (Dansham), inhibits rabbit platelet aggregation by suppressing intracellular calcium mobilization," Archives of Pharmacal Research, vol. 31, no. 1, pp. 47-53, 2008.

[30] P. Schäfer, M. Rodríguez, and G. Siegel, "Atherosclerosis, an inflammatory and fibroproliferative disease. A prophylactic phytochemical approach with Ginkgo biloba (EGb 761)," Atherosclerosis, vol. 195, no. 2, pp. 419-422, 2007.

[31] T. Hirase and K. Node, "Endothelial dysfunction as a cellular mechanism for vascular failure," The American Journal of Physiology-Heart and Circulatory Physiology, vol. 302, no. 3, pp. H499-H505, 2012.

[32] H. K. Saini, Y.-J. Xu, A. S. Arneja, P. S. Tappia, and N. S. Dhalla, "Pharmacological basis of different targets for the treatment of atherosclerosis," Journal of Cellular and Molecular Medicine, vol. 9, no. 4, pp. 818-839, 2005.

[33] H. Wang, X. Gao, and B. Zhang, "Tanshinone: an inhibitor of proliferation of vascular smooth muscle cells," Journal of Ethnopharmacology, vol. 99, no. 1, pp. 93-98, 2005.

[34] J.-R. Du, X. Li, R. Zhang, and Z.-M. Qian, “Tanshinone inhibits intimal hyperplasia in the ligated carotid artery in mice," Journal of Ethnopharmacology, vol. 98, no. 3, pp. 319-322, 2005.

[35] H.-J. Jin and C.-G. Li, "Tanshinone IIA and cryptotanshinone prevent mitochondrial dysfunction in hypoxia-induced H9c2 cells: association to mitochondrial ROS, intracellular nitric oxide, and calcium levels," Evidence-Based Complementary and Alternative Medicine, vol. 2013, Article ID 610694, 11 pages, 2013.

[36] H.-J. Jin, X.-L. Xie, J.-M. Ye, and C.-G. Li, “TanshinoneIIA and cryptotanshinone protect against hypoxia-induced mitochondrial apoptosis in H9c2 cells," PLoS ONE, vol. 8, no. 1, Article ID e51720, 2013. 
[37] I. T. Nizamutdinova, Y. M. Kim, H. Jin et al., "Tanshinone IIA inhibits TNF- $\alpha$-mediated induction of VCAM-1 but not ICAM1 through the regulation of GATA- 6 and IRF-1," International Immunopharmacology, vol. 14, no. 4, pp. 650-657, 2012.

[38] R. Lin, W.-R. Wang, J.-T. Liu, G.-D. Yang, and C.-J. Han, "Protective effect of tanshinone IIA on human umbilical vein endothelial cell injured by hydrogen peroxide and its mechanism," Journal of Ethnopharmacology, vol. 108, no. 2, pp. 217222, 2006.

[39] P. Chan, Y.-C. Chen, L.-J. Lin et al., “Tanshinone IIA attenuates $\mathrm{H}_{2} \mathrm{O}_{2}$-induced injury in human umbilical vein endothelial cells," The American Journal of Chinese Medicine, vol. 40, no. 6, pp. 1307-1319, 2012.

[40] C. Pan, L. Lou, Y. Huo et al., "Salvianolic acid B and Tanshinone IIA attenuate myocardial ischemia injury in mice by no production through multiple pathways," Therapeutic Advances in Cardiovascular Disease, vol. 5, no. 2, pp. 99-111, 2011.

[41] H.-S. Zhang and S.-Q. Wang, "Nrf2 is involved in the effect of tanshinone IIA on intracellular redox status in human aortic smooth muscle cells," Biochemical Pharmacology, vol. 73, no. 9, pp. 1358-1366, 2007.

[42] X. Li, J.-R. Du, Y. Yu, B. Bai, and X.-Y. Zheng, "Tanshinone IIA inhibits smooth muscle proliferation and intimal hyperplasia in the rat carotid balloon-injured model through inhibition of MAPK signaling pathway," Journal of Ethnopharmacology, vol. 129, no. 2, pp. 273-279, 2010.

[43] J. Gao, G. Yang, R. Pi et al., "Tanshinone IIA protects neonatal rat cardiomyocytes from adriamycin-induced apoptosis," Translational Research, vol. 151, no. 2, pp. 79-87, 2008.

[44] P. Yang, Y.-H. Jia, J. Li, L.-J. Li, and F.-H. Zhou, "Study of antimyocardial cell oxidative stress action and effect of tanshinone IIA on prohibitin expression," Journal of Traditional Chinese Medicine, vol. 30, no. 4, pp. 259-264, 2010.

[45] L. Zhang, Y. Wu, Y. Li et al., “Tanshinone IIA improves miR-133 expression through MAPK ERK1/2 pathway in hypoxic cardiac myocytes," Cellular Physiology and Biochemistry, vol. 30, no. 4, pp. 843-852, 2012.

[46] Y. Zhang, L. Zhang, W. Chu et al., "Tanshinone IIA inhibits miR-1 expression through 38 MAPK signal pathway in postinfarction rat cardiomyocytes," Cellular Physiology and Biochemistry, vol. 26, no. 6, pp. 991-998, 2010.

[47] X. Tan, J. Li, X. Wang et al., “Tanshinone IIA protects against cardiac hypertrophy via inhibiting cal-cineurin/Nfatc3 pathway," International Journal of Biological Sciences, vol. 7, no. 3, pp. 383-389, 2011.

[48] D.-X. Zhou, Q.-S. Liang, X.-X. He, and C.-Y. Zhan, "Changes of c-fos, c-jun mRNA expressions in cardiomyocyte hypertrophy induced by angiotensin II and effects of tanshinone II A," Zhongguo Zhong Yao Za Zhi, vol. 33, no. 8, pp. 936-939, 2008.

[49] H.-J. Hong, J.-C. Liu, T.-H. Cheng, and P. Chan, "Tanshinone IIA attenuates angiotensin II-induced apoptosis via Akt pathway in neonatal rat cardiomyocytes," Acta Pharmacologica Sinica, vol. 31, no. 12, pp. 1569-1575, 2010.

[50] D. Zhou, Z. Li, L. Zhang, and C. Zhan, "Inhibitory effect of tanshinone II A on TGF II- $\beta 1$-induced cardiac fibrosis," Journal of Huazhong University of Science and Technology [Medical Sciences], vol. 32, no. 6, pp. 829-833, 2012.

[51] P. Chan, J.-C. Liu, L.-J. Lin et al., "Tanshinone IIA inhibits angiotensin II-induced cell proliferation in rat cardiac fibroblasts," The American Journal of Chinese Medicine, vol. 39, no. 2, pp. 381394, 2011.
[52] L. Yang, X.-J. Zou, X. Gao et al., "Sodium tanshinone IIA sulfonate attenuates angiotensin II-induced collagen type I expression in cardiac fibroblasts in vitro," Experimental and Molecular Medicine, vol. 41, no. 7, pp. 508-516, 2009.

[53] P. Wang, S. Zhou, L. Xu et al., "Hydrogen peroxide-mediated oxidative stress and collagen synthesis in cardiac fibroblasts: blockade by tanshinone IIA," Journal of Ethnopharmacology, vol. 145, no. 1, pp. 152-161, 2013.

[54] S. Mao, W. Li, N. Qa'aty, M. Vincent, M. Zhang, and A. Hinek, "Tanshinone IIA inhibits angiotensin II induced extracellular matrix remodeling in human cardiac fibroblasts-implications for treatment of pathologic cardiac remodeling," International Journal of Cardiology, vol. 202, pp. 110-117, 2016.

[55] W.-Y. Wu, W.-Y. Wang, Y.-L. Ma et al., "Sodium tanshinone IIA silate inhibits oxygen-glucose deprivation/recovery-induced cardiomyocyte apoptosis via suppression of the NF- $\kappa \mathrm{B} / \mathrm{TNF}-$ $\alpha$ pathway," British Journal of Pharmacology, vol. 169, no. 5, pp. 1058-1071, 2013.

[56] T. Arino, K. Tanonaka, Y. Kawahara et al., "Effects of tanshinone VI on phosphorylation of ERK and Akt in isolated cardiomyocytes and cardiac fibroblasts," European Journal of Pharmacology, vol. 580, no. 3, pp. 298-305, 2008.

[57] X. Wang, Y. Wang, M. Jiang et al., "Differential cardioprotective effects of salvianolic acid and tanshinone on acute myocardial infarction are mediated by unique signaling pathways," Journal of Ethnopharmacology, vol. 135, no. 3, pp. 662-671, 2011.

[58] B. Wei, W.-W. Li, J. Ji, Q.-H. Hu, and H. Ji, “The cardioprotective effect of sodium tanshinone IIA sulfonate and the optimizing of therapeutic time window in myocardial ischemia/reperfusion injury in rats," Atherosclerosis, vol. 235, no. 2, pp. 318-327, 2014.

[59] B. Wei, M.-G. You, J.-J. Ling et al., "Regulation of antioxidant system, lipids and fatty acid $\beta$-oxidation contributes to the cardioprotective effect of sodium tanshinone IIA sulphonate in isoproterenol-induced myocardial infarction in rats," Atherosclerosis, vol. 230, no. 1, pp. 148-156, 2013.

[60] D. Sun, M. Shen, J. Li et al., "Cardioprotective effects of tanshinone IIA pretreatment via kinin B2 receptor-Akt-GSK-3 $\beta$ dependent pathway in experimental diabetic cardiomyopathy," Cardiovascular Diabetology, vol. 10, article 4, 2011.

[61] H. Hu, C. Zhai, G. Qian et al., "Protective effects of tanshinone IIA on myocardial ischemia reperfusion injury by reducing oxidative stress, HMGB1 expression, and inflammatory reaction," Pharmaceutical Biology, vol. 53, no. 12, pp. 1752-1758, 2015.

[62] M. Q. Zhang, Y. L. Zheng, H. Chen et al., "Sodium tanshinone IIA sulfonate protects rat myocardium against ischemiareperfusion injury via activation of PI3K/Akt/FOXO3A/Bim pathway," Acta Pharmacologica Sinica, vol. 34, no. 11, pp. 13861396, 2013.

[63] Y.-S. Li, Z.-H. Wang, and J. Wang, "Effect of tanshinone II A on angiotensin receptor in hypertrophic myocardium of rats with pressure over-loading," Zhongguo Zhong Xi Yi Jie He Za Zhi, vol. 28, no. 7, pp. 632-636, 2008.

[64] Y.-S. Li, Z.-H. Wang, L. Yan et al., "Effect of tashinone on nitric oxide synthase in hypertrophic cardiomyocyte of rats suffered abdominal aorta constriction," Zhongguo Zhong Yao Za Zhi, vol. 33, no. 12, pp. 1446-1450, 2008.

[65] E.-Y. Tu, Y.-G. Zhou, Z.-H. Wang, Q.-S. Liang, and G.-T. Yang, "Effects of tanshinone II A on the myocardial hypertrophy signal transduction system protein kinase B in rats," Chinese Journal of Integrative Medicine, vol. 15, no. 5, pp. 365-370, 2009. 
[66] Y. Li, Y. Yang, D. Yu, and Q. Liang, “The effect of tanshinone IIA upon the TGF-betal/Smads signaling pathway in hypertrophic myocardium of hypertensive rats," Journal of Huazhong University of Science and Technology [Medical Sciences], vol. 29, no. 4, pp. 476-480, 2009.

[67] J. Fang, S.-W. Xu, P. Wang et al., "Tanshinone II-A attenuates cardiac fibrosis and modulates collagen metabolism in rats with renovascular hypertension," Phytomedicine, vol. 18, no. 1, pp. 58-64, 2010.

[68] P. Wang, X. Wu, Y. Bao et al., “Tanshinone IIA prevents cardiac remodeling through attenuating $\mathrm{NAD}(\mathrm{P}) \mathrm{H}$ oxidase-derived reactive oxygen species production in hypertensive rats," Pharmazie, vol. 66, no. 7, pp. 517-524, 2011.

[69] H. Pang, B. Han, T. Yu, and Z. Peng, "The complex regulation of tanshinone IIA in rats with hypertension-induced left ventricular hypertrophy," PLoS ONE, vol. 9, no. 3, Article ID e92216, 2014.

[70] F. Tang, X. Wu, T. Wang et al., "Tanshinone II A attenuates atherosclerotic calcification in rat model by inhibition of oxidative stress," Vascular Pharmacology, vol. 46, no. 6, pp. 427-438, 2007.

[71] Z.-Y. Fang, R. Lin, B.-X. Yuan, G.-D. Yang, Y. Liu, and H. Zhang, "Tanshinone IIA downregulates the CD40 expression and decreases MMP-2 activity on atherosclerosis induced by high fatty diet in rabbit," Journal of Ethnopharmacology, vol. 115, no. 2, pp. 217-222, 2008.

[72] L. Huang, M. Zheng, Y. Zhou et al., "Tanshinone IIA attenuates cardiac dysfunction in endotoxin-induced septic mice via inhibition of NADPH oxidase 2-related signaling pathway," International Immunopharmacology, vol. 28, no. 1, pp. 444-449, 2015.

[73] K.-J. Huang, H. Wang, W.-Z. Xie, and H.-S. Zhang, "Investigation of the effect of tanshinone IIA on nitric oxide production in human vascular endothelial cells by fluorescence imaging," Spectrochimica Acta Part A: Molecular and Biomolecular Spectroscopy, vol. 68, no. 5, pp. 1180-1186, 2007.

[74] C.-C. Chang, C.-F. Chu, C.-N. Wang et al., "The antiatherosclerotic effect of tanshinone IIA is associated with the inhibition of TNF- $\alpha$-induced VCAM-1, ICAM-1 and CX3CL1 expression," Phytomedicine, vol. 21, no. 3, pp. 207-216, 2014.

[75] X. Liu, C. Y. Guo, X. J. Ma et al., "Anti-inflammatory effects of tanshinone IIA on atherosclerostic vessels of ovariectomized $\mathrm{ApoE}^{-1-}$ mice are mediated by estrogen receptor activation and through the ERK signaling pathway," Cellular Physiology and Biochemistry, vol. 35, no. 5, pp. 1744-1755, 2015.

[76] A. K. S. Wan, S. W. S. Leung, D.-Y. Zhu, and R. Y. K. Man, "Vascular effects of different lipophilic components of 'Danshen', a traditional Chinese medicine, in the isolated porcine coronary artery," Journal of Natural Products, vol. 71, no. 11, pp. 1825-1828, 2008.

[77] B. I. Jugdutt, "Ischemia/Infarction," Heart Failure Clinics, vol. 8, no. 1, pp. 43-51, 2012.

[78] P. Anversa, W. Cheng, Y. Liu, A. Leri, G. Redaelli, and J. Kajstura, "Apoptosis and myocardial infarction," Basic Research in Cardiology, vol. 93, supplement 3, pp. 8-12, 1998.

[79] A. Saraste, K. Pulkki, M. Kallajoki, K. Henriksen, M. Parvinen, and L.-M. Voipio-Pulkki, "Apoptosis in human acute myocardial infarction," Circulation, vol. 95, no. 2, pp. 320-323, 1997.

[80] L. M. Buja, "Myocardial ischemia and reperfusion injury," Cardiovascular Pathology, vol. 14, no. 4, pp. 170-175, 2005.
[81] E. Murphy and C. Steenbergen, "Mechanisms underlying acute protection from cardiac ischemia-reperfusion injury," Physiological Reviews, vol. 88, no. 2, pp. 581-609, 2008.

[82] A. P. Halestrap, S. J. Clarke, and S. A. Javadov, "Mitochondrial permeability transition pore opening during myocardial reperfusion-a target for cardioprotection," Cardiovascular Research, vol. 61, no. 3, pp. 372-385, 2004.

[83] D. Garcia-Dorado, M. Ruiz-Meana, and H. M. Piper, "Lethal reperfusion injury in acute myocardial infarction: facts and unresolved issues," Cardiovascular Research, vol. 83, no. 2, pp. 165-168, 2009.

[84] D. M. Yellon and D. J. Hausenloy, "Myocardial reperfusion injury," The New England Journal of Medicine, vol. 357, no. 11, pp. 1074-1135, 2007.

[85] Y.-I. Li, G. Elmer, and R. C. LeBoeuf, “Tanshinone IIA reduces macrophage death induced by hydrogen peroxide by upregulating glutathione peroxidase," Life Sciences, vol. 83, no. 15-16, pp. 557-562, 2008.

[86] S. Verma, P. W. M. Fedak, R. D. Weisel et al., "Fundamentals of reperfusion injury for the clinical cardiologist," Circulation, vol. 105, no. 20, pp. 2332-2336, 2002.

[87] M.-Q. Zhang, J.-F. Tu, H. Chen et al., "Janus kinase/signal transducer and activator of transcription inhibitors enhance the protective effect mediated by tanshinone IIA from hypoxic/ ischemic injury in cardiac myocytes," Molecular Medicine Reports, vol. 11, no. 4, pp. 3115-3121, 2015.

[88] W.-J. Zhang, J. Feng, R. Zhou et al., "Tanshinone IIA protects the human blood-brain barrier model from leukocyte-associated hypoxia-reoxygenation injury," European Journal of Pharmacology, vol. 648, no. 1-3, pp. 146-152, 2010.

[89] A. Yagi and S. Takeo, "Anti-inflammatory constituents, aloesin and aloemannan in Aloe species and effects of tanshinon VI in Salvia miltiorrhiza on heart," Yakugaku Zasshi, vol. 123, no. 7, pp. 517-532, 2003.

[90] B. C. Bernardo, K. L. Weeks, L. Pretorius, and J. R. McMullen, "Molecular distinction between physiological and pathological cardiac hypertrophy: experimental findings and therapeutic strategies," Pharmacology \& Therapeutics, vol. 128, no. 1, pp. 191$227,2010$.

[91] S. P. Barry, S. M. Davidson, and P. A. Townsend, "Molecular regulation of cardiac hypertrophy," International Journal of Biochemistry and Cell Biology, vol. 40, no. 10, pp. 2023-2039, 2008.

[92] A. Rohini, N. Agrawal, C. N. Koyani, and R. Singh, "Molecular targets and regulators of cardiac hypertrophy," Pharmacological Research, vol. 61, no. 4, pp. 269-280, 2010.

[93] P. M. Kang, P. Yue, Z. Liu, O. Tarnavski, N. Bodyak, and S. Izumo, "Alterations in apoptosis regulatory factors during hypertrophy and heart failure," American Journal of Physiology \& Heart and Circulatory Physiology, vol. 287, no. 1, pp. H72H80, 2004.

[94] E. Teiger, V. D. Than, L. Richard et al., "Apoptosis in pressure overload-induced heart hypertrophy in the rat," The Journal of Clinical Investigation, vol. 97, no. 12, pp. 2891-2897, 1996.

[95] V. P. M. van Empel and L. J. De Windt, "Myocyte hypertrophy and apoptosis: a balancing act," Cardiovascular Research, vol. 63, no. 3, pp. 487-499, 2004.

[96] M. Seddon, Y. H. Looi, and A. M. Shah, "Oxidative stress and redox signalling in cardiac hypertrophy and heart failure," Heart, vol. 93, no. 8, pp. 903-907, 2007. 
[97] F. Yang, P. Li, H. Li, Q. Shi, S. Li, and L. Zhao, "microRNA-29b mediates the antifibrotic effect of tanshinone IIA in postinfarct cardiac remodeling," Journal of Cardiovascular Pharmacology, vol. 65, no. 5, pp. 456-464, 2015.

[98] R. Jeyaseelan, C. Poizat, H.-Y. Wu, and L. Kedes, "Molecular mechanisms of doxorubicin-induced cardiomyopathy. Selective suppression of Reiske iron-sulfur protein, ADP/ATP translocase, and phosphofructokinase genes is associated with ATP depletion in rat cardiomyocytes," The Journal of Biological Chemistry, vol. 272, no. 9, pp. 5828-5832, 1997.

[99] Y.-W. Zhang, J. Shi, Y.-J. Li, and L. Wei, "Cardiomyocyte death in doxorubicin-induced cardiotoxicity," Archivum Immunologiae et Therapiae Experimentalis, vol. 57, no. 6, pp. 435-445, 2009.

[100] J. M. Berthiaume and K. B. Wallace, "Adriamycin-induced oxidative mitochondrial cardiotoxicity," Cell Biology and Toxicology, vol. 23, no. 1, pp. 15-25, 2007.

[101] A. R. Lehenbauer Ludke, A. A.-R. S. Al-Shudiefat, S. Dhingra, D. S. Jassal, and P. K. Singal, "A concise description of cardioprotective strategies in doxorubicin-induced cardiotoxicity," Canadian Journal of Physiology and Pharmacology, vol. 87, no. 10, pp. 756-763, 2009.

[102] X.-H. Tian and J. H. Wu, "Tanshinone derivatives: a patent review (January 2006-September 2012)," Expert Opinion on Therapeutic Patents, vol. 23, no. 1, pp. 19-29, 2013.

[103] Y.-F. Bi, H.-W. Xu, X.-Q. Liu, X.-J. Zhang, Z.-J. Wang, and H.M. Liu, "Synthesis and vasodilative activity of tanshinone IIA derivatives," Bioorganic and Medicinal Chemistry Letters, vol. 20, no. 16, pp. 4892-4894, 2010. 

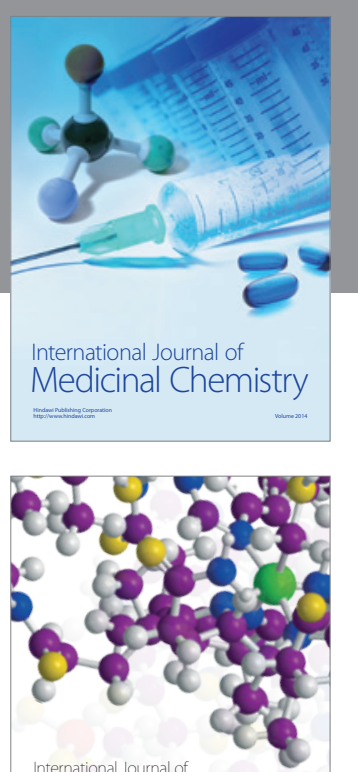

Carbohydrate Chemistry

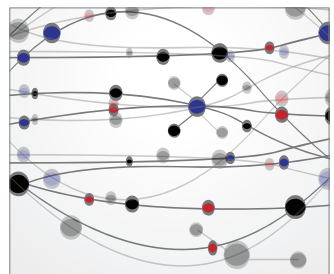

The Scientific World Journal
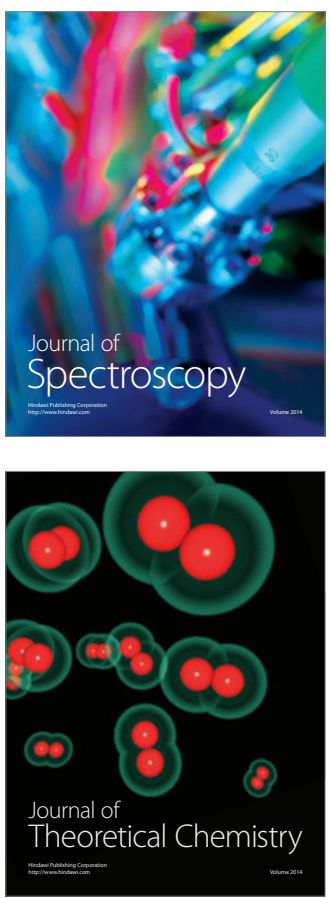
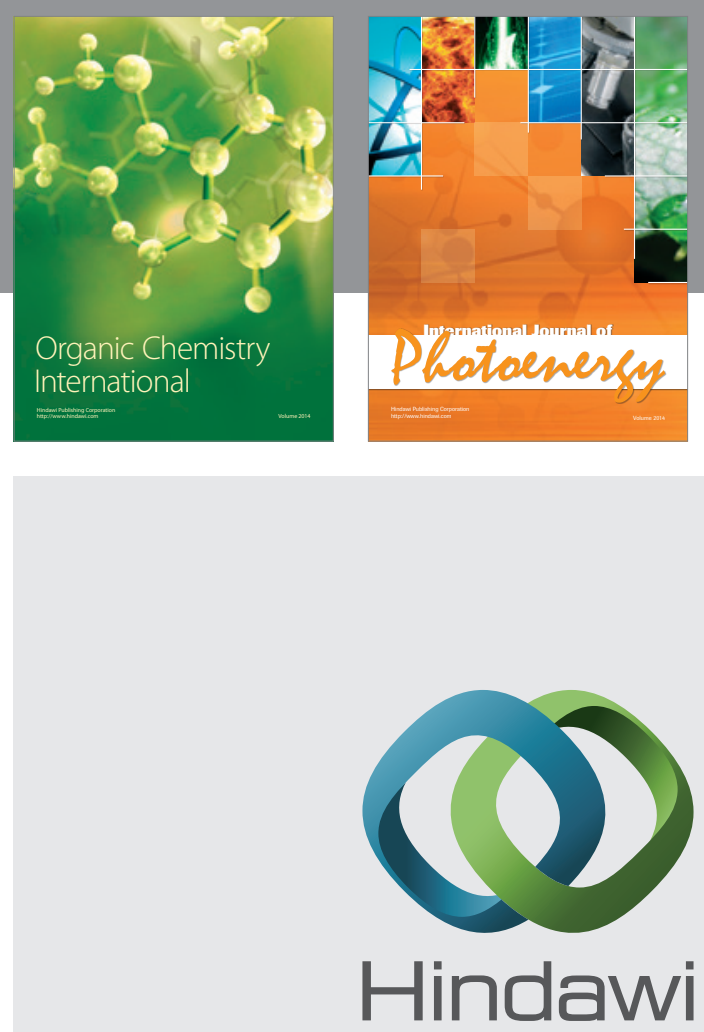

Submit your manuscripts at

http://www.hindawi.com

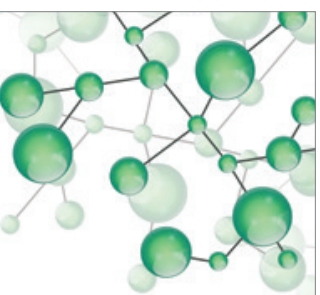

International Journal of

Inorganic Chemistry

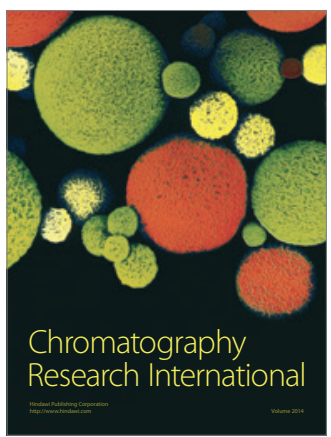

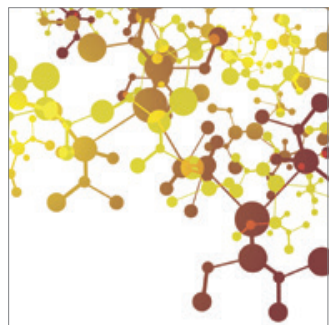

Applied Chemistry
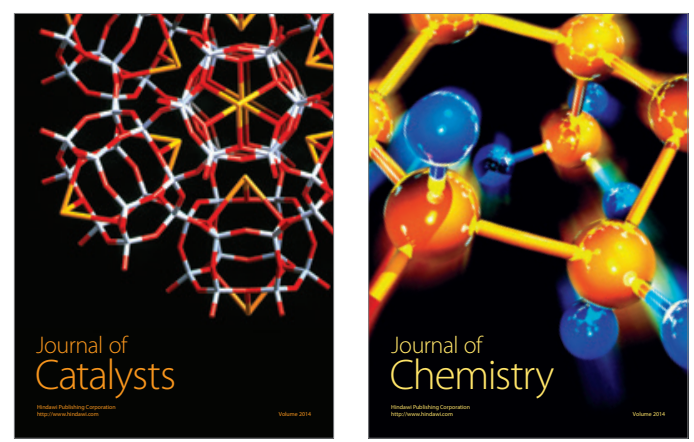
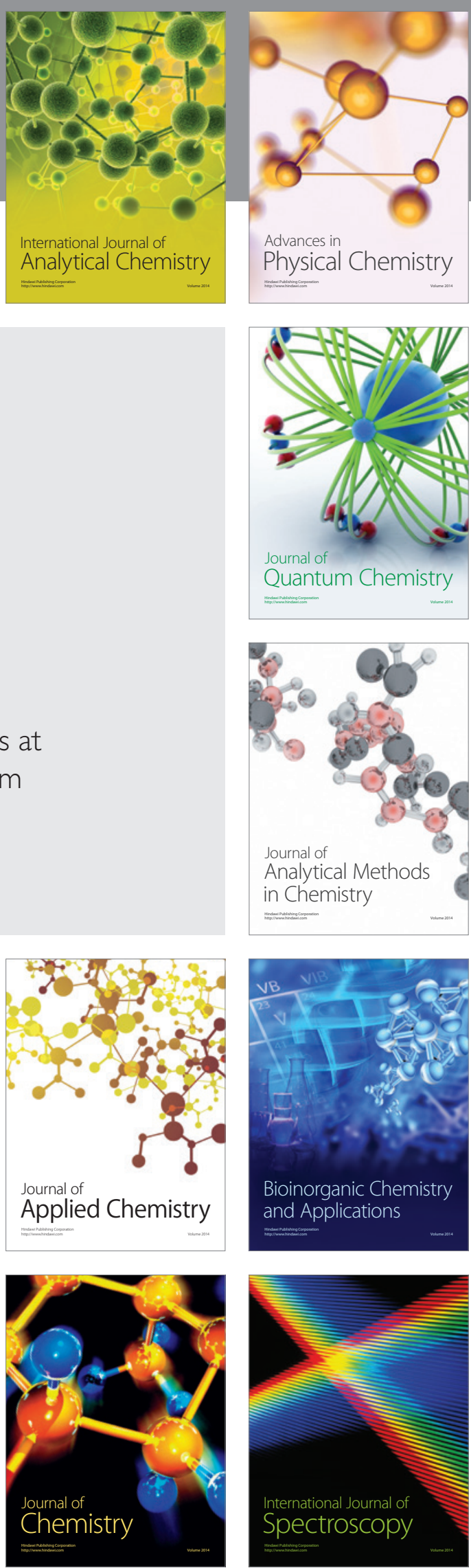